\title{
Pulmonary Thrombosis in COVID-19 Treated by Thrombolysis: A Small Case Series Using Streptokinase
}

\author{
Armando Caballero López, MD, PhD ${ }^{1}$ Carlos Herrera Cartaya, MD ${ }^{1}$ Elibet Chávez González, MD, PhD² \\ Delvis González Reinoso, $\mathrm{MD}^{3}$ Jorge Alain Caballero Font, $\mathrm{MD}^{1}$ Naivi Sánchez Vera, $\mathrm{MD}^{4}$ \\ Mario E. Nápoles Lizano, MD ${ }^{5}$ Alexander Vázquez Roque, MD ${ }^{6}$ Alexis Morales Valdera, MD $^{7}$ \\ Tania Crombet Ramos, MD, $\mathrm{PhD}^{8}$ Julio Betancourt Cervantes, MD, $\mathrm{PhD}^{9}$
}

${ }^{1}$ ICU Department, Arnaldo Milián Castro University Hospital, Santa Clara, Cuba

2 Electrophysiology Department, Cardiocenter Ernesto "Che” Guevara, Santa Clara, Cuba

${ }^{3}$ ICU Department, Manuel “Piti” Fajardo Rivero Hospital, Santa Clara, Cuba

${ }^{4}$ Clinical Laboratory, Manuel “Piti” Fajardo Rivero Hospital, Santa Clara, Cuba

${ }^{5}$ Radiology Department, Cardiocenter Ernesto "Che" Guevara, Santa Clara, Cuba

${ }^{6}$ Radiology Department, Celestino Hernández Robau University Hospital, Santa Clara, Cuba

${ }^{7}$ Internal Medicine, International Clinic of North Keys, Villa Clara, Cuba

${ }^{8}$ Clinical Immunology Division, Center for Molecular Immunology (CIM), Havana, Cuba

${ }^{9}$ General Surgery Department, Manuel “Piti” Fajardo Rivero Hospital, Santa Clara, Cuba

Semin Thromb Hemost 2021;47:431-435.

Cuba has been affected by coronavirus disease 2019 (COVID19) with more than 2,400 cases diagnosed, 87 deaths, and $3.55 \%$ lethality, by July $17,2020 .^{1}$ COVID- 19 is a gradually evolving pathology, characterized by a series of stages sustained by different molecular and biological mechanisms. The disease can be divided in at least five different phases: incubation, respiratory, proinflammatory, prothrombotic, and death or remission. ${ }^{2}$

COVID-19 may predispose patients to thrombotic disease, both in venous and arterial circulation, due to excessive inflammation, endothelial dysfunction, platelet activation, and many other prothrombotic factors such as tissue injury, decrease of natural anticoagulants, and stasis. ${ }^{3}$ Despite the use of antiviral, anti-inflammatory drugs, anticoagulant therapy, and even invasive mechanical ventilation, many patients progress to a critical stage, characterized by acute respiratory distress syndrome (ARDS) and shock. ${ }^{4}$ We describe here two patients, in whom thrombolysis using re-

published online December 30, 2020
Issue Theme Maintaining Hemostasis and Preventing Thrombosis in COVID-19 -Part II; Guest Editors: Emmanuel J. Favaloro, PhD, FFSc (RCPA) and Giuseppe Lippi, MD.

Address for correspondence Tania Crombet Ramos, MD, PhD, Center of Molecular Immunology, Ave 216, Esq. 15. Atabey, Playa, Havana, P.O. Box 16040, Cuba (e-mail: taniac@cim.sld.cu).

combinant streptokinase ${ }^{5}$ was used to treat refractory hypoxemia after suspecting pulmonary micro- and macrothrombotic disease.

Patient 1: A 53-year-old woman with previous history of hypertension and type 2 diabetes mellitus was admitted at the intensive care unit (ICU) after moderate hypoxemia and respiratory alkalosis. Her initial oxygenation index $\left(\mathrm{PaO}_{2} /\right.$ $\mathrm{FiO}_{2}$ ) was 191 . Diagnosis of severe acute respiratory syndrome coronavirus 2 (SARS-CoV-2) infection was confirmed with real time polymerase chain reaction (RT-PCR) on a nasopharyngeal sample. Upon admission, she was receiving treatment with lopinavir/ritonavir, chloroquine, interferon $\alpha$, and ceftriaxone. She developed several CovID-19 complications including nosocomial respiratory infection (Pseudomonas aeruginosa, Staphylococcus aureus, and Candida albicans), requiring endotracheal intubation and mechanical ventilation. Chest X-rays showed interstitial lesions in both lung fields (matching with stage II of physiopathology). She

(c) 2020. Thieme. All rights reserved. Thieme Medical Publishers, Inc., 333 Seventh Avenue, 18th Floor, New York, NY 10001, USA
DOI https://doi.org/ 10.1055/s-0040-1716872. ISSN 0094-6176. 
was hence treated with the anti-CD6 antibody itolizumab, ${ }^{6}$ which significantly reduced interleukin-6 serum concentration. Patient experienced radiological and clinical improvement, though definitive ventilator weaning was not possible. After three unsuccessful extubation attempts in 15 days, radiological and clinical deterioration developed, including hypotension requiring dobutamine administration at a dose of $20 \mu \mathrm{g} / \mathrm{kg} / \mathrm{min}$. A computer tomography (CT) scan showed ground glass images at both lung fields ( - Fig. 1A, B). D-dimer was $2.32 \mu \mathrm{g} / \mathrm{mL}, \mathrm{PaO}_{2} / \mathrm{FiO}_{2}$ decreased to 100 , the alveolararterial oxygen gradient $\left[\mathrm{D}(\mathrm{A}-\mathrm{a}) \mathrm{O}_{2}\right]$ was 483 , pulmonary shunt fraction (Qs/Qt) ratio was $57.9 \%$, the arterial-venous $\mathrm{O}_{2}$ content difference $\left(\mathrm{Ca}-\mathrm{vO}_{2}\right)$ was 5.14 , and the positive end-expiratory pressure (PEEP) was $8 \mathrm{~cm}$ of water. After the patient respiratory worsening together with the increase in the D-dimer, the ICU's expert committee decided to start thrombolysis with recombinant streptokinase ${ }^{7}$ at a dose of $250,000 \mathrm{IU}$ in the first hour followed by $100,000 \mathrm{IU}$ every hour for 24 hours. Streptokinase infusion was only administered for 6 hours due to oral cavity bleeding. Hemorrhage was rapidly controlled after stopping infusion. The use of tranexamic acid was not required. In spite of the premature streptokinase discontinuation, D-dimer values decreased to $0.7 \mu \mathrm{g} / \mathrm{mL}$ (D-dimer units), $\mathrm{PaO}_{2} / \mathrm{FiO}_{2}$ raised to 410 , blood pressure normalized, and dobutamine could be withdrawn. In parallel, alternative prone (12-16 hours) and supine ventilation ( 8 hours) was established. After thrombolysis, the fraction of inspired oxygen was reduced $\left(\mathrm{FiO}_{2}=0.4\right)$ and ventilation parameters began to improve: $\mathrm{PaO}_{2}$ was $164 \mathrm{~mm} \mathrm{Hg}$ and oxygen saturation reached 99.2\%. Patient also showed a remarkable clinical and radiological improvement (-Fig. 1C). Twenty-four hours later, the prone and supine ventilation continued to alternate, the attendance rate of the assistance mechanical ventilator was gradually reduced to $30 \%$, PEEP was decreased to $5 \mathrm{~cm}$ of water, and $\mathrm{FiO}_{2}$ to 0.30 . In the next 5 days, complete ventilator weaning was achieved. A third CT scan was performed 10 days after the thrombolysis ( - Fig. 1D), showing sustained radiological improvement. After 39 days in ICU, the patient was transferred to hospital conventional ward to continue pulmonary and neuromuscular rehabilitation.

Patient 2: An 86-year-old man with a personal history of essential hypertension, generalized atherosclerosis, and cognitive disorder, was admitted at the "Celestino Hernandez Robau" University Hospital of Santa Clara. Upon admission, RT-PCR for SARS-CoV-2 was positive on a nasopharyngeal sample and the patient showed polypnea (32 rotations per minute) and ancillary respiratory musculature use for breathing. His blood pressure was $90 / 60 \mathrm{~mm} \mathrm{Hg}$. He was hence treated according the Cuban national protocol

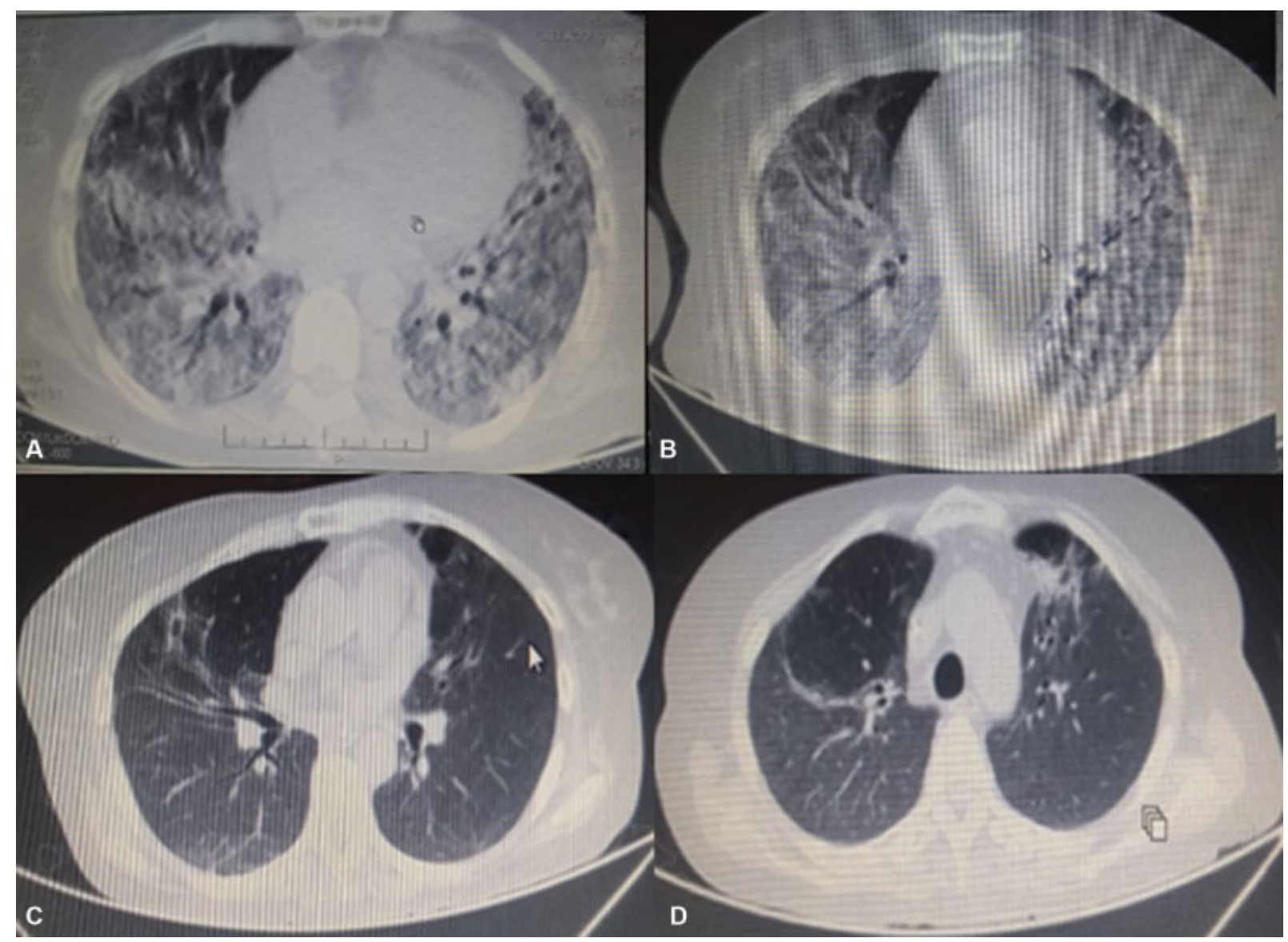

Fig. 1 Patient 1. (A, B) Multislice computed tomography (CT) scan before thrombolysis: Ground glass images are observed in both lung fields, with small areas of hyperlucency toward the vertex. (C) Multislice CT image performed 48 hours after thrombolysis, with clear tomographic improvement. (D) Multislice CT image performed 7 days after thrombolysis, where the tomographic improvement becomes more evident. 


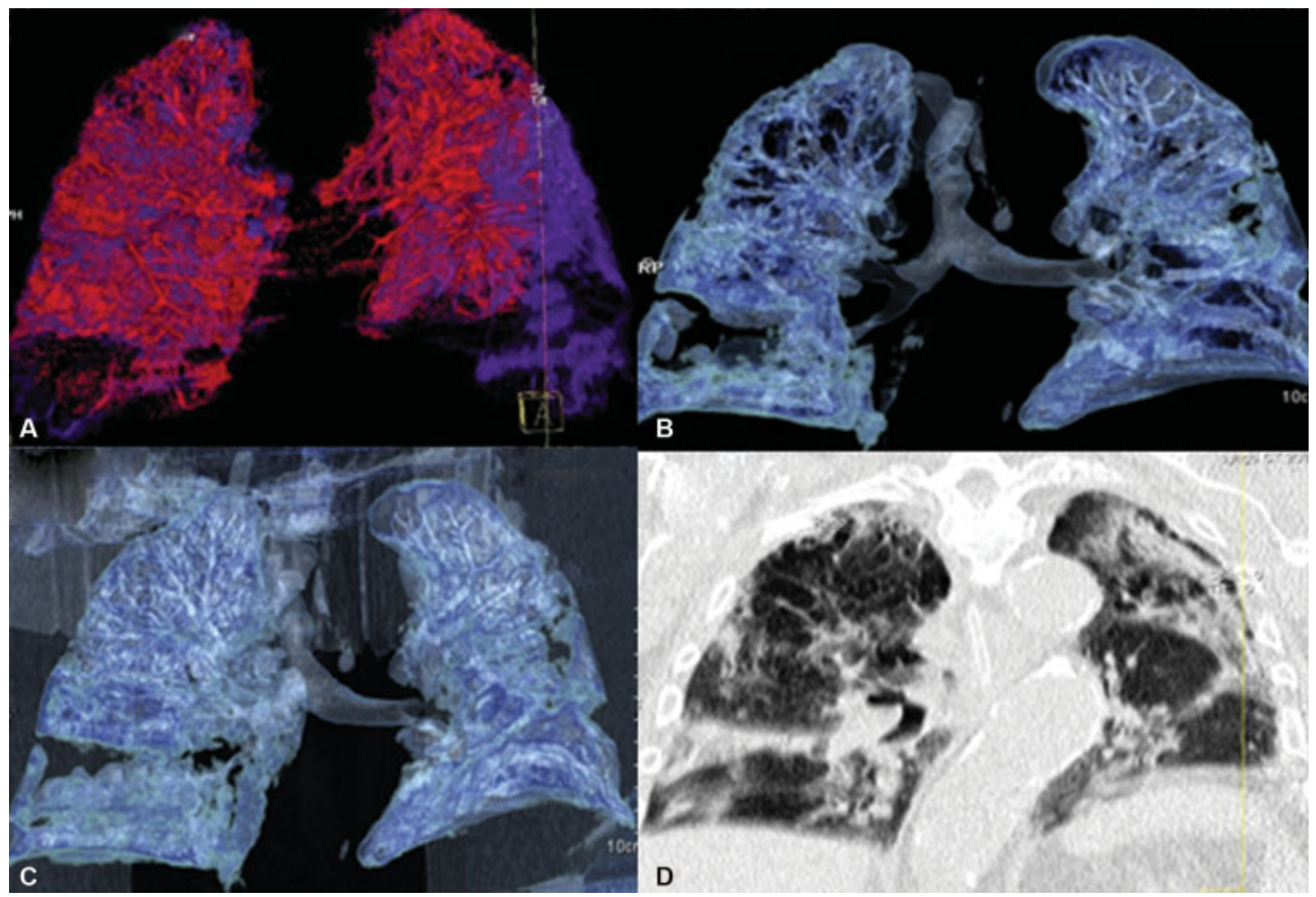

Fig. 2 Patient 2. (A) Multislice computerized axial tomography (120 cuts), with dual energy tomographic views, showing a zone of pulmonary infarction (black) at the base of the right and left lung. Thrombus zone with laterobasal ischemia of the left lung (violet) and multiple and small areas of purplish color, pulmonary microthrombi, in both lung fields predominate on the right side. (B) Anterior view of multiplanar airways, with black areas of pulmonary infarcts and diffuse inflammatory lesions of both lung bases. (C) Another anterior view of axial tomography computed with dual energy, with pulmonary infarcts (black) and areas of diffuse inflammatory lesions predominantly of the lower lobes. (D) Computerized axial tomography, shaggy aorta with diffuse atheromatosis in all segments of the aorta.

including chloroquine, lopinavir/ritonavir, recombinant erythropoietin (EPO) (cytoprotective dose), and low molecular weight heparin (fraxiparine), at 0.6 IU every 12 hours. EPO was included in the protocol for COVID-19 considering its cytoprotective capacity including anti-ischemic, antiapoptotic, and regenerative effect in several tissues such as the lung, kidney, heart, nervous system, retina, pancreas, and endothelial cells. EPO acts through a special receptor, EPOR$\beta c R$, which mediated its protective effects in critically ill patients and following trauma. ${ }^{8}$ Patient also received CIGB258, ${ }^{9}$ a peptide with immune-regulatory properties, as part of an expanded access protocol. Despite treatment, patient suffered COVID-19 complications including nosocomial respiratory infection (Pseudomonas aeruginosa was isolated in blood culture and bronchial secretions) requiring antibiotic therapy, endotracheal intubation, and mechanical ventilation. The initial evaluation of the arterial blood gases showed severe hypoxemia with $\mathrm{PaO}_{2} / \mathrm{FiO}_{2}$ of 68 . Chest X-rays showed interstitial lesions in both lung fields. Ventilator was used in volume-control mode, PEEP was adjusted to $10 \mathrm{~cm}$ of water, and $\mathrm{FiO}_{2}$ was 0.60 . Urine output gradually decreased, creatinine reached $224 \mu \mathrm{mol} / \mathrm{mL}$, glomerular filtration rate was $20.59 \mathrm{~mL} / \mathrm{min}$, and patient showed signs of hypovolemia. Due to these hemodynamic changes, treatment with norepinephrine $(0.2 \mu \mathrm{g} / \mathrm{kg} / \mathrm{min})$ and dobutamine $(10 \mu \mathrm{g} / \mathrm{kg} /$ min) was initiated. Patient also had radiologic and respiratory worsening, including tracheobronchial secretions and leukocytosis of $18.0 \times 10^{9} / \mathrm{L}$. X-rays showed triangular images at the base of the right lung suggestive of pulmonary

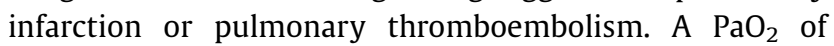
$58 \mathrm{~mm} \mathrm{Hg}$ and $\mathrm{PaO}_{2} / \mathrm{FiO}_{2}$ of 96 , forced to keep $\mathrm{FiO}_{2}$ high (0.8) and to start maneuvers of alveolar recruitment. PEEP was increased to $12 \mathrm{~cm}$ of water, CIGB-258 dose augmented, and alternating prone and supine ventilation started. As a result, oxygen saturation improved to 98 to $99 \%$. However, after an elevated D-dimer ( $8 \mu \mathrm{g} / \mathrm{mL}$; D-dimer units), arterial hypotension, $\mathrm{D}(\mathrm{A}-\mathrm{a}) \mathrm{O}_{2}$ of 486 , and Qs/Qt of $57 \%$ together with a significant clinical-radiological worsening, thrombolysis was proposed as therapeutic alternative. At that point, a confirmatory CT scan of the pulmonary thrombosis or microthrombosis was not feasible, due to the patient's extreme poor condition. Thrombolysis with recombinant streptokinase $^{5}$ at 250,000 IU for 1 hour followed by 100,000 IU every 1 hour for 24 hours was planned. Streptokinase administration was interrupted due to onset of oral and endotracheal bleeding that was rapidly contained with tranexamic acid $(1.0 \mathrm{~g})$. Shortly after and even though the full streptokinase dose was not administered, a remarkable clinical and radiological improvement occurred; $\mathrm{PaO}_{2}$ increased to $79.2 \mathrm{~mm}$ $\mathrm{Hg}$ and $\mathrm{PaO}_{2} / \mathrm{FiO}_{2}$ raised to 158 . Seventy-two hours after 
thrombolysis, a CT scan (120-slice) combined with a dual energy CT scan, was performed (-Fig. 2). Patient subsequently had negative RT-PCR for SARS-CoV-2 on a nasopharyngeal sample, 15 days after admission. CT scan showed areas of pulmonary infarcts, microthrombi, and diffuse inflammatory lesions of both lung bases. After initial improvement, a persistent multidrug-resistant Pseudomonas aeruginosa was isolated from the respiratory secretion. At that time, he had received therapy with meronem, colistina, and piperacillin-tazobactam. Patient had progressive radiological and clinical worsening associated with severe bronchopneumonia. The patient remained ventilated and unresponsive to any of the antibiotic schemes used. He died 40 days after his admission in the hospital and 25 days after thrombolysis. Death was attributed to septic shock. Necropsy was not performed.

Patients admitted to the ICU for COVID-19, following an infection by the SARS-CoV-2, have a considerably increased risk of thrombotic events, both arterial and venous. ${ }^{4}$

SARS-CoV-1 and -2 show tropism for angiotensin-converting enzyme 2 on type II pneumocytes. ${ }^{10}$ This tropism, along with the close anatomical juxtaposition of type II pneumocytes and pulmonary vascular network, together with a severe inflammatory reaction, is likely to drive the generalized pulmonary hypercoagulable state seen in patients with COVID-19. ${ }^{10}$ Development of hypoxemia, secondary to ARDS induced by COVID-19 might also activate the coagulation cascade and could be important in endothelial dysfunction beyond the capillary network. ${ }^{3}$

Although the number of postmortem pathologic reports are limited, Luo et al described vascular wall thickening, stenosis of the vascular lumen, and microthrombus formation accompanying the findings of ARDS. ${ }^{11}$ Similar pathologic findings are found in small vessels of other organs. ${ }^{12-14}$ Ackermann et al reported that alveolar capillary microthrombi were more prevalent in patients with COVID-19 than in patients with influenza. ${ }^{15}$ The frequency of venous thromboembolism in critically ill patients with COVID-19, including deep vein thrombosis and pulmonary embolism, is becoming better understood, ranging from 25 to $27 \%$ in recent publications. ${ }^{16,17}$ Besides these pathology findings, many physicians have reported D-dimer elevation in COVID19 patients, mainly in those bearing severe and critical disease. ${ }^{18}$ Although D-dimer is not a specific indicator of thrombosis, in combination with other parameters, its elevation may suggest a systemic coagulation activation with increased thrombin generation and fibrinolysis. ${ }^{19}$

Here, we present the results of the first two Cuban patients carrying COVID-19, in whom thrombolysis was attempted (considering their extreme severity, their refractory hypoxemia, and high suspicion of micro- and macrothrombosis).

A few reports on thrombolysis for COVID-19, using tissue plasminogen activator as a thrombolytic agent, have been published. ${ }^{20}$ Our two patients were treated with recombinant streptokinase, which has been the thrombolytic of choice to treat coronary thrombosis and pulmonary or peripheral venous macrothrombi in Cuba. ${ }^{5,7}$ Both patients had hemorrhagic complications that precluded administering the full dose. Other thrombolytic agents are unavailable in Cuba.

Confirming multiple pulmonary microthrombi is challenging. In COVID-19, clinical and laboratory manifestations can raise the index of diagnostic suspicion. A thoracic angiotomography can confirm a diagnosis of pulmonary thromboembolism of thick or medium branches or multiple pulmonary microthrombi disseminated in both lung fields. A multislice CT with dual energy has the potential to improve the detection of pulmonary thrombosis. ${ }^{21}$

We hence conclude that the two patients took some advantages from the thrombolysis, since this therapeutic strategy was effective to improve their respiratory function and chest images. Unfortunately, one patient died due to septic respiratory complications. In both cases, rescue thrombolysis was done on compassionate basis after interdisciplinary discussion. We advise, however, that this procedure should not be done routinely unless in the setting of a clinical trial.

Bleeding occurred in both cases but was controlled after stopping infusion or with the use of tranexamic acid. We therefore believe that thrombolysis might be a valuable alternative for COVID-19 patients with pulmonary microor macrothrombosis that have failed anti-inflammatory and anticoagulant therapy. Nevertheless, the effectiveness of thrombolysis for COVID-19 patients should be confirmed in randomized clinical trials.

\section{Conflict of Interest}

None declared.

\section{Acknowledgments}

We are extremely grateful to other ICU physicians, nurses, and general staff that also took care of these two critically ill COVID-19 patients, who stayed in the hospital for more than 40 days.

\section{References}

1 Ministerio de Salud Pública de Cuba Revised on June 30, 2020. Available at: https://salud.msp.gob.cu/category/covid-19/. Accessed September 2, 2020

2 Lippi G, Sanchis-Gomar F, Henry BM. COVID-19: unravelling the clinical progression of nature's virtually perfect biological weapon. Ann Transl Med 2020;8(11):693

3 Harenberg J, Favaloro E. COVID-19: progression of disease and intravascular coagulation - present status and future perspectives. Clin Chem Lab Med 2020;58(07):1029-1036

4 Klok FA, Kruip MJHA, van der Meer NJM, et al. Incidence of thrombotic complications in critically ill ICU patients with COVID-19. Thromb Res 2020;191:145-147

5 Llerena LD, Cáceres-Lóriga FM, Betancourt BY. Recombinant streptokinase: evidences from clinical use. Eur Heart J 2005;26 (14):1448-1449, author reply 1449-1450

6 Bughani U, Saha A, Kuriakose A, et al. T cell activation and differentiation is modulated by a CD6 domain 1 antibody Itolizumab. PLoS One 2017;12(07):e0180088

7 Betancourt BY, Marrero-Miragaya MA, Jiménez-López GCuban National Network of Pharmacoepidemiology. , et al; . Pharmacovigilance program to monitor adverse reactions of recombinant 
streptokinase in acute myocardial infarction. BMC Clin Pharmacol 2005;5:5

8 Hadadi A, Mortezazadeh M, Kolahdouzan K, Alavian G. Does recombinant human erythropoietin administration in critically ill COVID-19 patients have miraculous therapeutic effects? J Med Virol 2020;92(07):915-918

9 Corrales O, Hernández L, Prada D, et al. CIGB-814, an altered peptide ligand derived from human heat-shock protein 60 , decreases anti-cyclic citrullinated peptides antibodies in patients with rheumatoid arthritis. Clin Rheumatol 2019;38 (03):955-960

10 Rivellese F, Prediletto E. ACE2 at the centre of COVID-19 from paucisymptomatic infections to severe pneumonia. Autoimmun Rev 2020;19(06):102536

11 Luo W, Yu H, Gou J, et al. Clinical pathology of critical patient with novel coronavirus pneumonia (COVID-19). Preprints 2020; 2020:2020020407

$12 \mathrm{Li} \mathrm{T}$, Lu H, Zhang W. Clinical observation and management of COVID-19 patients. Emerg Microbes Infect 2020;9(01):687-690

13 Zhang Y, Cao W, Xiao M, et al. Clinical and coagulation characteristics of 7 patients with critical COVID-2019 pneumonia and acroischemia [in Chinese]. Zhonghua Xue Ye Xue Za Zhi 2020;41(04): 302-307
14 Zhou M, Zhang X, Qu J. Coronavirus disease 2019 (COVID-19): a clinical update. Front Med 2020;14(02):126-135

15 Ackermann M, Verleden SE, Kuehnel M, et al. Pulmonary vascular endothelialitis, thrombosis, and angiogenesis in Covid-19. N Engl J Med 2020;383(02):120-128

16 Delabranche X, Helms J, Meziani F. Immunohaemostasis: a new view on haemostasis during sepsis. Ann Intensive Care 2017;7 (01):117

17 Maatman TK, Jalali F, Feizpour C, et al. Routine venous thromboembolism prophylaxis may be inadequate in the hypercoagulable state of severe coronavirus disease 2019. Crit Care Med 2020;48 (09):e783-e790

18 Lippi G, Favaloro EJ. D-dimer is associated with severity of coronavirus disease 2019: a pooled analysis. Thromb Haemost 2020;120(05):876-878

19 Thachil J, Tang N, Gando S, et al. Laboratory haemostasis monitoring in COVID-19. J Thromb Haemost 2020

20 Poor HD, Ventetuolo CE, Tolbert T, et al. COVID-19 critical illness pathophysiology driven by diffuse pulmonary thrombi and pulmonary endothelial dysfunction responsive to thrombolysis. Clin Transl Med 2020

21 Lu GM, Wu SY, Yeh BM, Zhang LJ. Dual-energy computed tomography in pulmonary embolism. Br J Radiol 2010;83(992):707-718 